\title{
Role of PD-L1 Expression during the Progression of Submucosal Gastric Cancer
}

\author{
Yasunari Ubukata ${ }^{a}$ Kyoichi Ogata ${ }^{a}$ Makoto Sohda ${ }^{a}$ Takehiko Yokoboria \\ Yuki Shimoda $^{b}$ Tadashi Handa $^{b}$ Nobuhiro Nakazawa ${ }^{a} \quad$ Akiharu Kimura $^{a}$ \\ Norimichi Kogure $^{a}$ Akihiko Sano ${ }^{a}$ Makoto Sakai ${ }^{a}$ Hiroomi Ogawa ${ }^{a}$ \\ Hiroyuki Kuwano $^{a}$ Ken Shirabe $^{a}$ Tetsunari Oyama $^{b}$ Hiroshi Saeki $^{a}$ \\ aDepartment of General Surgery, Graduate School of Medical Sciences, Gunma University, Maebashi, Japan; \\ ${ }^{b}$ Department of Pathology, Graduate School of Medical Sciences, Gunma University, Maebashi, Japan
}

\section{Keywords}

Early gastric cancer · Tumor invasion - Lamina muscularis mucosa $\cdot$ Invasive front $\cdot$ Immune checkpoint proteins

\begin{abstract}
Introduction: Programmed death-ligand 1 (PD-L1) expression is a prognostic marker for gastric cancer that correlates with tumor diameter and depth of penetration. But the role of PD-L1 and mechanism(s) employed in the initial phase of invasion in early gastric cancer is yet to be understood. Objective: This study aims to elucidate the role of PD-L1 during the progression of gastric cancer, specifically invading the submucosa beyond the lamina muscularis mucosa. Methods: Using 107 patients with pathological submucosal gastric cancer, we determined the expression of PD-L1 based on the staining of the cell membrane or cytoplasm of tumor cells in the central and invasive front of the tumor. Samples were categorized into 3 groups based on the intensity of PD-L1 expression. $\mathrm{CD}^{+}$lymphocytes expressing PD- 1 and $\mathrm{CD} 163^{+}$macrophages were used to determine the number of cell nuclei at the invasive front, similar to PD-L1. CMTM6 levels were deter-
\end{abstract}

mined and used to stratify samples into 3 groups. Results: PD-L1 expression was higher in the invasive front $(26.2 \%)$ than in the central portion of the tumors $(7.4 \% ; p<0.001)$. Moreover, lymphatic and vascular invasion were more frequently observed in samples with high levels of PD-L1 (lymphatic invasion: 60.7 vs. $35.4 \%, p=0.0026$, and vascular invasion: 39.3 vs. $16.5 \%, p=0.0018$ ). There was no correlation between PD-L1 expression and the levels of PD-1, CD8, CD163, and CMTM6. Conclusions: PD-L1-expressing cancer cells at the invasive front of gastric cancer influence the initial stages of tumor invasion and lymphovascular permeation in earlystage gastric cancers. Immune checkpoint signaling may be the driving force in the invasive front during the invasion of the submucosa beyond the lamina muscularis mucosa.

(c) 2020 S. Karger AG, Basel

\section{Introduction}

Although there has been a gradual decrease in the rate of mortality associated with gastric cancer, it remains the third most common cause of cancer-related deaths in Ja-

karger@karger.com
www.karger.com/ocl
Karger ${ }^{\prime /}$

Makoto Sohda

Department of General Surgery, Graduate School of Medical Sciences Gunma University

3-39-15, Showa-machi, Maebashi, Gunma 371-8511 (Japan)

msohda@gunma-u.ac.jp 
pan, claiming 45,000 lives annually [1]. Although gastric cancer does not usually involve venous and lymphatic permeation among the mucosal cancers, upon progression into the submucosa, patients develop lymph node and other distant metastases [2]. However, the progression of mucosal cancer into the submucosal region remains to be elucidated. Therefore, it is important to understand the mechanism(s) involved during the initial stages of invasion in gastric cancer.

Venous and lymphatic permeation is attributed to the penetration of cancer cells deeper into the mucosa and submucosa during the progression of gastric cancer [3]. Several reports have shown that clinicopathological findings, such as tumor diameter, vascular invasion, degree of differentiation, and depth of penetration, help track the progress of lymph node metastasis [4-8]. Depth of penetration includes the extent of cancer invasion; this can be determined by analyzing the gene expression of claudin 3 and claudin 18 owing to their involvement in the initial phase of gastric cancer invasion $[9,10]$. However, there is little mechanistic insight into the role with deep invasion during the early stages of gastric cancer.

$\mathrm{PD}-\mathrm{L} 1 / 2$ is found on the surface of cancer cells that bind to the immune checkpoint receptor PD-1 on the surface of cytotoxic $T$ cells, thereby inactivating active $T$ cells and evading immune recognition [11-13]. Immune checkpoint inhibitors targeting PD-1/PD-L1 suppress $\mathrm{T}$ cell inactivation and activate tumor immunity $[14,15]$. PD-L1 is also involved in tumor invasion; Tsutsumi et al. [16] reported the correlation between PD-L1 expression and poor prognosis of patients with esophageal cancer along with epithelial-mesenchymal transition. Numerous other studies have shown that PD-L1 expression correlates with poor prognoses for various malignant tumors, such as esophageal cancers, hepatocellular cancers, renal cell cancers, and malignant melanomas [17-20]. The expression of PD-L1, high content of infiltrated lymphocytes in tumors, and distant metastasis in breast cancer can be attributed to the levels of PD-1 [21].

$\mathrm{PD}-\mathrm{L} 1$ expression is a prognostic marker for gastric cancer that correlates with tumor diameter and depth of penetration [22]. Li et al. [23] demonstrated a decrease in tumor metastasis and proliferation by the knocking down of PD-L1 in gastric cancer cell lines. PD-L1 expression in gastric cancer is controlled by IFN- $\gamma$ via JAK-STAT signaling and epithelial-mesenchymal transition; PD-L1 affects tumor immunity by modulating cytotoxic T lymphocyte activity [24]. However, the role of PD-L1 and mechanism(s) employed in the initial phase of invasion in early gastric cancer is yet to be understood. We hypoth- esize that PD-L1 may contribute to the acquisition of invasive phenotypes of cancer cells during progression from the mucosal layer to the stromal cells-abundant submucosal layer, as it theoretically acts in immune evasion. This study aims to elucidate the role of PD-L1 during gastric cancer progression, specifically in the invasion of the submucosa beyond the lamina muscularis mucosa. Thus, we determined the expression of PD-L1 in different regions of submucosal gastric cancer. Subsequently, we investigated the correlation between PD-L1 expression, CMTM6 (in the regulation of PD-L1), and infiltration of immune cells (e.g., tumor-associated $\mathrm{CD}_{163^{+}}$macrophages and cytotoxic $\mathrm{CD} 8^{+} \mathrm{T}$ lymphocytes expressing PD-1) in gastric cancer tissues.

\section{Materials and Methods}

\section{Patients}

We performed radical surgeries on 635 gastric cancer patients at the Gunma University Hospital between April 2010 and March 2017. Among them, 107 patients who underwent surgery for primary gastric cancer with pT1b (pathological results showing tumor cell infiltration in the submucosal layer through the mucosal muscle plate) were included. Among pT1b carcinomas, pSM1 (pT1b1) is defined by a distance from the lower end of the mucosal muscle plate to the deepest point of $<500 \mu \mathrm{m}$, and pSM2 (pT1b2) is defined by a distance of $>500 \mu \mathrm{m}$. This study was approved by the Ethics Committee of Gunma University (approval No.: HS2019-183).

\section{Immunohistochemistry}

Biomarker expression was analyzed by immunohistochemistry. Briefly, 4- $\mu \mathrm{m}$ sections were deparaffinized in xylene and dehydrated using a gradient of ethanol. The specimens were pretreated in an autoclave to retrieve the following antigens: PD-L1 and PD-1 $\left(121^{\circ} \mathrm{C}, 10 \mathrm{~min}\right.$, Target Retrieval Solution; $\left.\mathrm{pH} 6.1\right), \mathrm{CD} 8\left(121^{\circ} \mathrm{C}\right.$, 30 min, boiled citric acid; pH 6.0), CD163, CMTM6, and D2-40 $\left(98^{\circ} \mathrm{C}, 30 \mathrm{~min}\right.$, boiled citric acid; $\left.\mathrm{pH} 6.0\right)$. Subsequently, the sections were incubated in $0.3 \%$ hydrogen peroxide dissolved in methanol to saturate and inhibit the endogenous peroxidases. Nonspecific binding by antibodies was prevented by blocking the sections followed by overnight incubation at room temperature with primary antibodies against PD-L1 (1:400; 28-8, Abcam), PD-1 (1:400; NAT105, Abcam), CD8 (1:100; C8/144B, DAKO), CD163 (1:500; D6U1J, Cell Signaling Technology), CMTM6 (1:50; ab97652, Abcam), and D2-40 (1:200; M3619, DAKO).

The sections were washed with phosphate-buffered saline, and staining by the primary antibodies was visualized using the Histofine Simple Stain MAX-PO (Multi) Kit (Nichirei, Tokyo, Japan) according to the instructional manual. The chromogen $(0.02 \%)$, 3,3-diaminobenzidine tetrahydrochloride (Dojindo Laboratories, Kumamoto, Japan), was dissolved in $0.005 \% \mathrm{H}_{2} \mathrm{O}_{2}$ and $50 \mathrm{mM}$ Tris- $\mathrm{HCl}$ buffer ( $\mathrm{pH}$ 7.6). Subsequently, the sections were lightly counterstained with Mayer's hematoxylin and mounted.

PD-L1, PD-1, CD8, CD163, and CMTM6 levels were independently determined by 2 investigators, including 1 general patholo-
16

Oncology 2021;99:15-22 DOI: $10.1159 / 000509033$
Ubukata et al. 

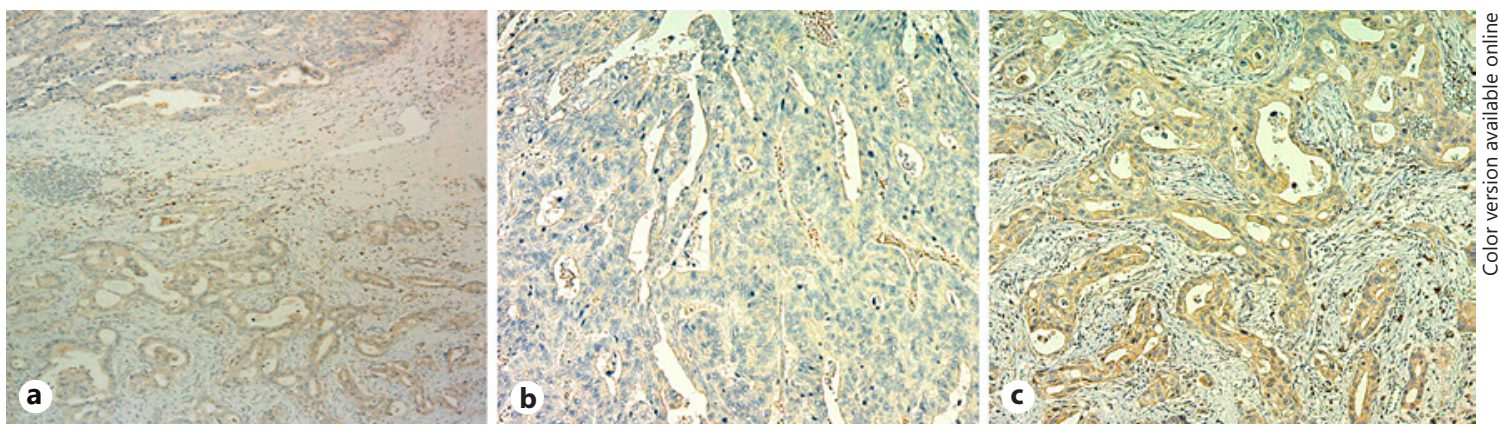

Fig. 1. Expression of PD-L1 in the central, invasive, and submucosal regions of gastric carcinoma. Representative immunohistochemistry images of samples stained with anti-PD-L1 antibodies $(\times 100$, $\mathbf{a}$ : central portion; $\mathbf{b}$ : invasive front; and $\times 50$, c: submucosal region).

gist. PD-L1 expression was analyzed based on the staining of the cell membrane or cytoplasm of tumor cells in the central and invasive tumor samples. The immunostaining score was calculated based on the number of positive cells and staining intensity, as described previously [25]. Samples with PD-L1 expression were categorized into 3 groups (no, low, and high expression) based on the Allred score [26]. PD-1, CD8, and CD163 expressions were analyzed using the number of positive cell nuclei at the invasive front of the tumor (similar to PD-L1), as described previously [27, 28]. CMTM6 expression was analyzed as per a previous study [29].

Clinicopathological Features and PD-L1 Expression in Patients

The patients were stratified in 2 different ways. First, we assigned patients to no/low expression and high expression groups based on PD-L1 expression at the invasive portion of the tumor. Second, we assigned patients to the center-dominant (stronger or equal expression of PD-L1 in the central rather than invasive front) and invasive front-dominant (stronger PD-L1 expression in the invasive front rather than the central portion) groups. Clinicopathological features (such as age, gender, differentiation of gastric cancer, tumor size, pathological lymph node metastasis, lymphatic permeation, venous permeation, postoperative recurrence, and cancer death) were comparatively analyzed in each subgroup.

\section{Statistical Analysis}

The differences between the 2 groups were compared using Student's $t$ test and the $\chi^{2}$ test. $p$ values $<0.05$ were considered statistically significant. All statistical analyses were performed using EZR (2.4-0). Standard deviations, instead of standard errors of the mean, were preferred. All significant results necessarily included the test value, degree(s) of freedom, and probability level.

\section{Results}

\section{PD-L1 Expression in the Central and Invasive Portion} of Submucosal Gastric Cancer

Table 1 shows the immunohistochemical profile of the central and invasive portions of the tumor. PD-L1 was expressed in the cell membrane and cytoplasm of tumor
Table 1. Comparison of PD-L1 expression between the central and invasive front portions in resected specimens of submucosal gastric cancer

\begin{tabular}{lccl}
\hline PD-L1 expression & $\begin{array}{c}\text { Central } \\
\text { portion }\end{array}$ & $\begin{array}{l}\text { Invasive } \\
\text { front portion }\end{array}$ & $\begin{array}{l}p \\
\text { value }\end{array}$ \\
\hline All cases $(n=107)$ & & & \\
$\quad$ No expression & $57(53.3)$ & $10(9.3)$ & $<0.001$ \\
Low expression & $42(39.3)$ & $69(64.5)$ & \\
High expression & $8(7.4)$ & $28(26.2)$ & \\
pSM1 cases $(n=24)$ & & & \\
No expression & $13(54.2)$ & $2(8.3)$ & 0.002 \\
Low expression & $9(37.5)$ & $18(75)$ & \\
High expression & $2(8.3)$ & $4(16.7)$ & \\
pSM2 cases $(n=83)$ & & & \\
No expression & $44(53)$ & $8(9.6)$ & $<0.001$ \\
Low expression & $33(39.3)$ & $51(61.5)$ & \\
High expression & $6(7.2)$ & $24(28.9)$ & \\
\hline
\end{tabular}

The numbers in parentheses indicate percentages. PD-L1, programmed death-ligand 1.

cells in the submucosal gastric cancer specimens (Fig. 1a, b). Among the 107 patient samples, PD-L1 was observed to be higher in the invasive front $(26.2 \%)$ than in the central portion $(7.4 \% ; p<0.001)$. Similarly, in the pathological SM1 and pathological SM2 subgroups, we observed a higher expression of PD-L1 in the invasive front than in the central portion (pSM1: 16.7 vs. $8.3 \%, p=0.002$; pSM2: 28.9 vs. $7.2 \%, p<0.001)$.

\section{Clinicopathological Features of the Patient Cohort Based on PD-L1 Expression}

Table 2 summarizes the clinicopathological features based on the expression of PD-L1 in the invasive front of the gastric tumors. Samples with high PD-L1 expression 
Table 2. Clinicopathological features of patients with submucosal gastric cancer who underwent gastrectomy relative to $\mathrm{PD}-\mathrm{L} 1$ expression at the invasive front portion

\begin{tabular}{|c|c|c|c|}
\hline \multirow[t]{2}{*}{ Factor } & \multicolumn{2}{|c|}{ PD-L1 expression } & \multirow{2}{*}{$\begin{array}{l}p \\
\text { value }\end{array}$} \\
\hline & $\begin{array}{l}\text { no }+ \text { low } \\
(n=79)\end{array}$ & $\begin{array}{l}\text { high } \\
(n=28)\end{array}$ & \\
\hline Mean age, years & 69.0 & 69.0 & 0.949 \\
\hline \multicolumn{4}{|l|}{ Gender } \\
\hline Male & $57(72.2)$ & $19(67.9)$ & \multirow[t]{2}{*}{0.809} \\
\hline Female & $22(17.8)$ & $9(22.1)$ & \\
\hline \multicolumn{4}{|c|}{ Differentiation of gastric cancer } \\
\hline Well/moderately & $63(79.7)$ & $21(75.0)$ & \multirow[t]{2}{*}{0.588} \\
\hline Poorly & $16(20.3)$ & $7(25.0)$ & \\
\hline Mean tumor size, $\mathrm{mm}$ & 33 & 34.5 & 0.507 \\
\hline \multicolumn{4}{|c|}{ Pathological lymph node metastasis } \\
\hline$(-)$ & $66(83.5)$ & $22(78.6)$ & \multirow[t]{2}{*}{0.570} \\
\hline$(+)$ & $13(16.5)$ & $6(22.4)$ & \\
\hline \multicolumn{4}{|l|}{ Lymphatic permeation } \\
\hline$(-)$ & $51(64.6)$ & $11(39.3)$ & \multirow[t]{2}{*}{0.026} \\
\hline$(+)$ & $28(35.4)$ & $17(60.7)$ & \\
\hline \multicolumn{4}{|l|}{ Venous permeation } \\
\hline$(-)$ & $66(83.5)$ & $17(60.7)$ & \multirow[t]{2}{*}{0.018} \\
\hline$(+)$ & $13(16.5)$ & $11(39.3)$ & \\
\hline \multicolumn{4}{|c|}{ Postoperative recurrence } \\
\hline$(-)$ & $74(93.7)$ & $28(100)$ & \multirow[t]{2}{*}{0.323} \\
\hline$(+)$ & $5(6.3)$ & 0 & \\
\hline \multicolumn{4}{|l|}{ Cancer death } \\
\hline$(-)$ & 75 (94.9) & $28(100)$ & \multirow[t]{2}{*}{0.571} \\
\hline$(+)$ & $4(5.1)$ & 0 & \\
\hline
\end{tabular}

The numbers in parentheses indicate percentages. PD-L1, programmed death-ligand 1 . correlated with a higher proportion of lymphatic and vascular invasion (lymphatic invasion: 60.7 vs. $35.4 \%, p=$ 0.0026 , and vascular invasion: 39.3 vs. $16.5 \%, p=0.0018$ ). There were no significant differences in patient age and gender, tumor size and differentiation, lymph node metastases, postoperative recurrence, and cancer death between the 2 groups.

\section{Clinicopathological Features of the Patient Cohort}

Based on the Dominant Pattern of PD-L1 Expression

Table 3 shows the clinicopathological features based on the dominant pattern of PD-L1 expression. Pathological lymph node metastasis and lymphatic and vascular invasion were more frequently observed in the invasive front-dominant group (pathological lymph node metastasis: 25.4 vs. $6.8 \%, p=0.019$; lymphatic invasion: 52.4 vs. $27.3 \%, p=0.011$; and vascular invasion: 30.2 vs. $11.4 \%$, $p=0.033)$. There were no significant differences in pa-
Table 3. Clinicopathological features of patients with submucosal gastric cancer who underwent gastrectomy relative to the dominant pattern of PD-L1 expression

\begin{tabular}{|c|c|c|c|}
\hline \multirow[t]{2}{*}{ Factor } & \multicolumn{2}{|c|}{ PD-L1 expression pattern } & \multirow{2}{*}{$\begin{array}{l}p \\
\text { value }\end{array}$} \\
\hline & $\begin{array}{l}\text { center } \\
\text { dominant } \\
(n=44)\end{array}$ & $\begin{array}{l}\text { invasive front } \\
\text { dominant } \\
(n=63)\end{array}$ & \\
\hline Mean age, years & 70 & 68 & 0.316 \\
\hline Gender & $28(63.6)$ & $48(76.2)$ & 0.196 \\
\hline Male & $16(36.4)$ & $15(23.8)$ & \\
\hline \multicolumn{4}{|l|}{ Female } \\
\hline \multicolumn{4}{|c|}{ Differentiation of gastric cancer } \\
\hline Well/moderately & $30(68.2)$ & $54(85.7)$ & \multirow[t]{2}{*}{0.035} \\
\hline Poorly & $14(31.8)$ & $9(14.3)$ & \\
\hline Mean tumor size, $\mathrm{mm}$ & 35 & 33 & 0.653 \\
\hline \multicolumn{4}{|c|}{ Pathological lymph node metastasis } \\
\hline$(-)$ & $41(93.2)$ & $47(74.6)$ & \multirow[t]{2}{*}{0.019} \\
\hline$(+)$ & $3(6.8)$ & $16(25.4)$ & \\
\hline \multicolumn{4}{|l|}{ Lymphatic permeation } \\
\hline$(-)$ & $32(72.7)$ & $30(47.6)$ & \multirow[t]{2}{*}{0.011} \\
\hline$(+)$ & $12(27.3)$ & $33(52.4)$ & \\
\hline \multicolumn{4}{|l|}{ Venous permeation } \\
\hline$(-)$ & $39(88.6)$ & $44(69.8)$ & \multirow[t]{2}{*}{0.033} \\
\hline$(+)$ & $5(11.4)$ & $19(30.2)$ & \\
\hline \multicolumn{4}{|l|}{ Postoperative recurrent } \\
\hline$(-)$ & $43(97.7)$ & $59(93.7)$ & \multirow[t]{2}{*}{0.647} \\
\hline$(+)$ & $1(2.3)$ & $4(6.3)$ & \\
\hline \multicolumn{4}{|l|}{ Cancer death } \\
\hline$(-)$ & $44(100)$ & $59(93.7)$ & \multirow[t]{2}{*}{0.142} \\
\hline$(+)$ & 0 & $4(6.3)$ & \\
\hline
\end{tabular}

The numbers in parentheses indicate percentages. PD-L1, programmed death-ligand 1.

tient age, gender, tumor size, differentiation, postoperative recurrence, and cancer death between the 2 groups.

\section{Correlation between PD-L1 Expression and Other \\ Factors}

We determined the expression of PD-1, CD8, CD163, and CMTM6 in the invasive front of the submucosal tumors and compared them with PD-L1 expression (Fig. 2). We used 2 different ways of subgrouping and found that there was no significant correlation between PD-L1 expression and other patient factors in either subgroup (PD-1: $p=0.37$; CD8: $p=0.39$; CD163: $p=0.6$; and CMTM6: $p=0.23$; Table 4 ).

\section{PD-L1 and D2-40 Profiles in Samples with Lymphatic} Permeation

We performed PD-L1 and D2-40 staining using serial sections of the representative patient sample with lym- 
Fig. 2. Expression of PD-1, CD8, CD163, and CMTM6 at the invasive front of the tumor. Representative immunohistochemistry images of samples stained with antiPD-1 ( $\times 200, \mathbf{a})$, anti-CD8 $(\times 200, \mathbf{b})$, antiCD163 $(\times 200, \mathbf{c})$, and anti-CMTM6 $(\times 200$, d) antibodies.
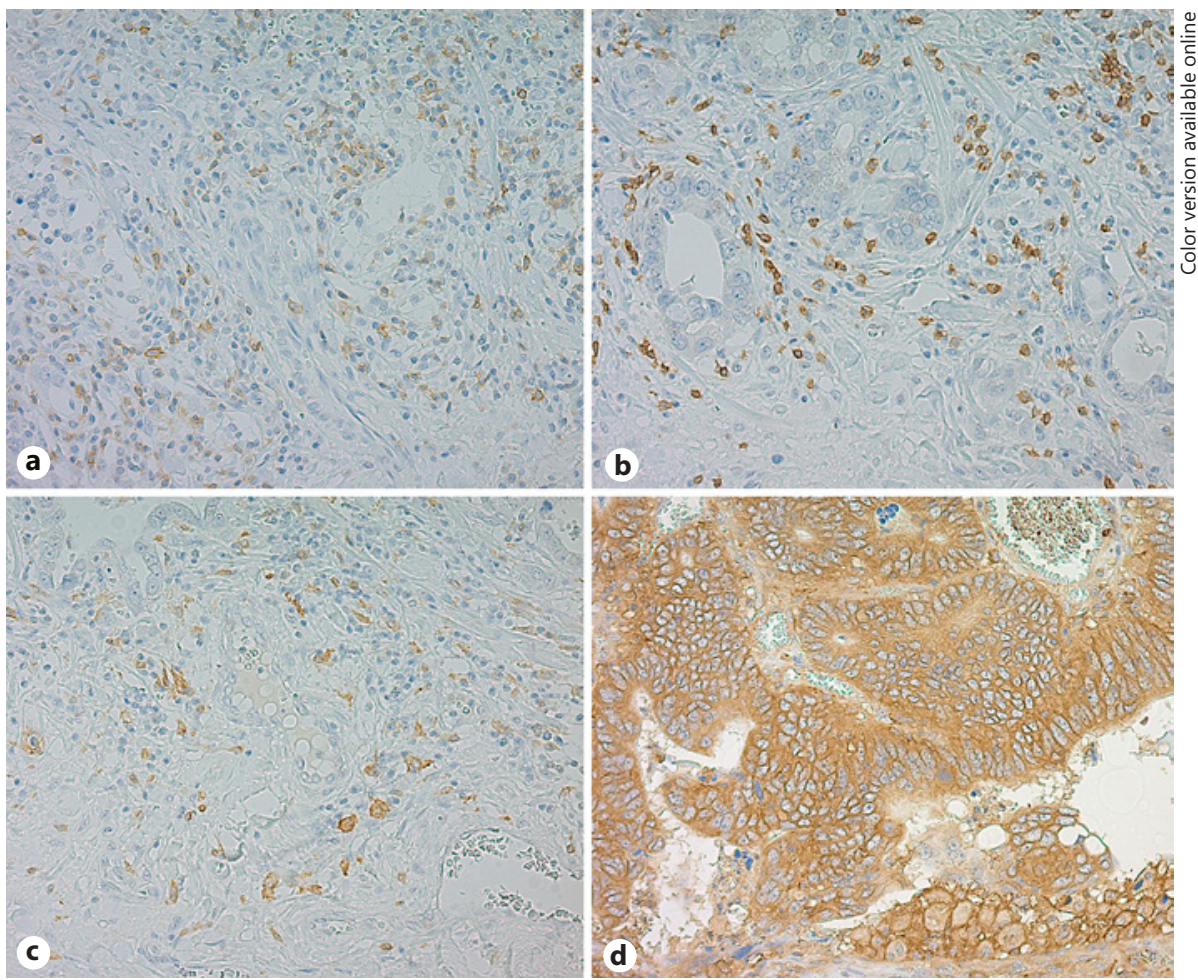

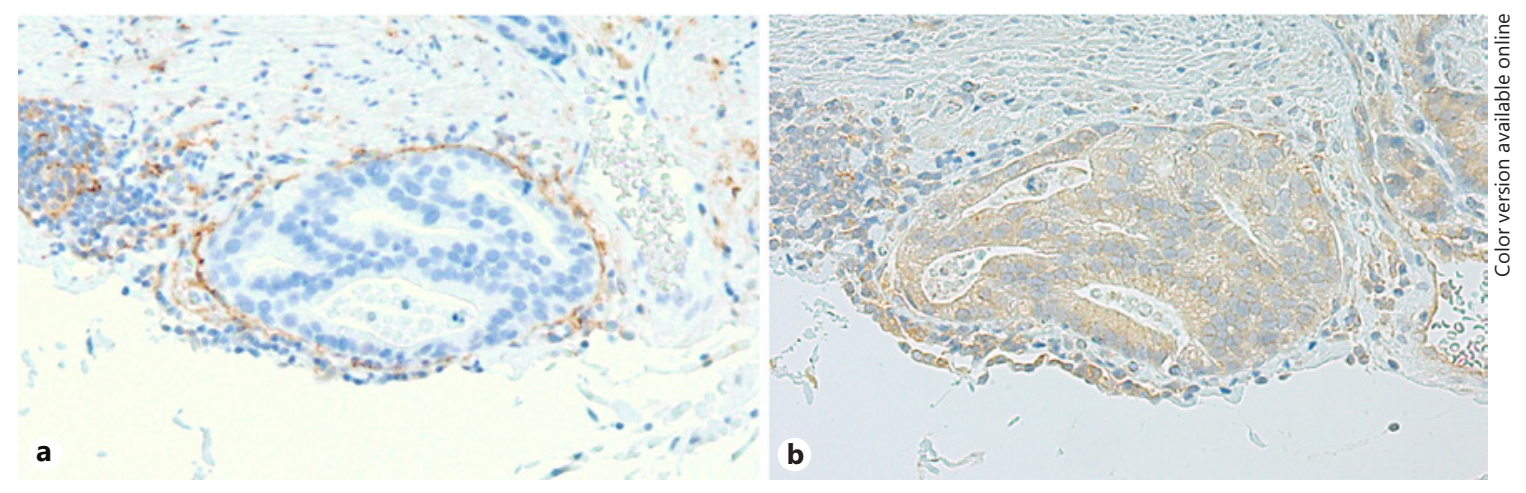

Fig. 3. Expression of PD-L1 in the tumor cells present in the lymphatic vessel at the invasive front of the submucosal gastric cancer. Representative immunohistochemistry images of samples stained with D2-40 $(\times 400$, a) and anti-PD-L1 $(\times 400, \mathbf{b})$ antibodies.

phatic permeation (Fig. 3). PD-L1 was expressed in the cancer cells that permeated into lymphatic vessels.

\section{Discussion/Conclusion}

Cancer cells that penetrate the lamina muscularis mucosa and invade into the submucosa (with an abundance of stromal cells) are exposed to host immune response. Therefore, we hypothesized that PD-L1-expressing can- cer cells at the invasive front escape immune recognition and infiltrate deep into the host tissues to enhance tumor progression. In this study, we observed high levels of $\mathrm{PD}$ L1 within the PSM1 subgroup, indicating the importance of PD-L1 in the initial stages of gastric cancer invasiveness.

Venous and lymphatic permeation occurs as cancers progress, thereby enabling metastasis to the lymph nodes and distant organs. Akt, mTOR, VEGF-C, and VEGF-D are also involved during this process [30]. In this study, 
Table 4. Relationship of expression between PD-L1 and other factors relative to $\mathrm{PD}-\mathrm{L} 1$ expression at the invasive front portion and the dominant pattern of PD-L1 expression in resected specimens of submucosal gastric cancers

\begin{tabular}{|c|c|c|c|}
\hline \multirow[t]{2}{*}{ Factor } & \multicolumn{2}{|c|}{$\begin{array}{l}\text { PD-L1 expression at invasive } \\
\text { front portion }\end{array}$} & \multirow[t]{2}{*}{$\begin{array}{l}p \\
\text { value }\end{array}$} \\
\hline & $\begin{array}{l}\text { no }+ \text { low } \\
(n=79)\end{array}$ & $\begin{array}{l}\text { high } \\
(n=28)\end{array}$ & \\
\hline \multicolumn{4}{|l|}{ PD-1 } \\
\hline Negative & $53(67.1)$ & $16(57.1)$ & \multirow[t]{2}{*}{0.37} \\
\hline Positive & $26(22.9)$ & $12(42.9)$ & \\
\hline \multicolumn{4}{|l|}{ CD8 } \\
\hline Negative & $36(45.6)$ & $10(35.7)$ & \multirow[t]{2}{*}{0.39} \\
\hline Positive & $43(54.4)$ & $18(64.3)$ & \\
\hline \multicolumn{4}{|l|}{ CD163 } \\
\hline Negative & $63(79.7)$ & $21(75.0)$ & \multirow[t]{2}{*}{0.6} \\
\hline Positive & $16(20.3)$ & $7(25.0)$ & \\
\hline \multicolumn{4}{|l|}{ CMTM6 } \\
\hline Negative & $24(30.4)$ & $5(17.9)$ & \multirow[t]{2}{*}{0.23} \\
\hline Positive & $55(69.6)$ & $23(82.1)$ & \\
\hline \multirow[t]{2}{*}{ Factor } & \multicolumn{2}{|c|}{ PD-L1 expression pattern } & \multirow{2}{*}{$\begin{array}{l}p \\
\text { value }\end{array}$} \\
\hline & $\begin{array}{l}\text { center } \\
\text { dominant } \\
(n=44)\end{array}$ & $\begin{array}{l}\text { invasive front } \\
\text { dominant } \\
(n=63)\end{array}$ & \\
\hline \multicolumn{4}{|l|}{ PD-1 } \\
\hline Negative & $31(70.5)$ & $38(60.3)$ & \multirow[t]{2}{*}{0.31} \\
\hline Positive & 13 (29.5) & 25 (39.7) & \\
\hline \multicolumn{4}{|l|}{ CD8 } \\
\hline Negative & $22(50)$ & $24(38.1)$ & \multirow[t]{2}{*}{0.24} \\
\hline Positive & $22(50)$ & 39 (61.9) & \\
\hline \multicolumn{4}{|l|}{ CD163 } \\
\hline Negative & $35(79.5)$ & 49 (77.8) & \multirow[t]{2}{*}{1} \\
\hline Positive & $9(20.5)$ & $14(22.2)$ & \\
\hline \multicolumn{4}{|l|}{ CMTM6 } \\
\hline Negative & $16(36.4)$ & $13(20.6)$ & \multirow[t]{2}{*}{0.081} \\
\hline Positive & $28(63.6)$ & $50(79.4)$ & \\
\hline
\end{tabular}

The numbers in parentheses indicate percentages. PD-L1, programmed death-ligand 1; PD-1, programmed death 1; CMTM6, CKLF-like MARVEL transmembrane domain-containing 6.

we found that PD-L1 expression correlated with venous and lymphatic permeation in submucosal gastric cancer samples. PD-L1 and D2-40 staining of the tissue sections of a representative patient with lymphatic permeation revealed the presence of PD-L1 expression in cancer cells that permeated into the lymphatic vessels (Fig. 3). These findings suggest that cancer cells expressing a high content of PD-L1 in submucosal invasive cancers have the ability of deep invasion and vascular permeation.
$\mathrm{CD}^{+} \mathrm{T}$ cells influence tumor immunity by differentiating into cytotoxic $\mathrm{T}$ lymphocytes. CD8 cell infiltration has been shown to correlate with PD-L1 expression in tumor cells and stroma $[30,31]$. CD8 ${ }^{+} \mathrm{T}$ cell infiltration correlates with $\mathrm{PD}-\mathrm{L} 1$ expression and poor prognosis in gastric cancer [32]. CD163 is expressed on the surface of M2 macrophages; M2 macrophages stimulate the formation of the tumor microenvironment and early vascular invasion in gastric cancer [33]. CD163 expression on M2 macrophages has been reported to correlate with PD-L1 expression in gastric cancer. PD- 1 is expressed on $\mathrm{CD} 4^{+}$and $\mathrm{CD}^{+} \mathrm{T}$ cells and interacts with PD-L1/2 in avoiding immune responses. Burr et al. [34] reported that CMTM6 stabilizes PD-L1 from degradation by ubiquitination. In this study, we did not observe a correlation between the expression of PD-L1 in cancer cells and that in other proteins. Shen et al. [35] revealed that there was no correlation with CD8 and PD-L1, suggesting that an adaptive immune resistance mechanism may be inactive in earlystage cancer. Okabe et al. [36] reported that $\mathrm{CD}^{+} \mathrm{T}$ cell density was not associated with PD-L1 expression in early breast cancer. Concerning the relationship between PDL1 and CD163, to our knowledge, no previous study has focused on early-stage cancer. The studies that examined the relationship between PD-L1 and CD163 in gastric [28] and colorectal cancers [37] showed a positive correlation between PD-L1 and CD163; probably, this could be attributed to the use of samples with mixed stages of cancer (mainly T2 or deeper cancers). We speculated that tumor microenvironment might be immature in $\mathrm{pT} 1 \mathrm{~b}$ gastric cancer regardless of high $\mathrm{PD}-\mathrm{L} 1$ expression on cancer cells, compared to advanced cancer. Therefore, it is important to understand the interaction between the tumor and stroma in the future, especially in early-stage cancer.

This study demonstrated that PD-L1 was highly expressed at the invasive front of submucosal invasive gastric cancer, indicating heterogeneous expression of PD-L1 in gastric cancer. However, biopsies from patients in a clinical trial that studied the effect of anti-PD-1 as a therapy against advanced gastric cancer revealed that PD-L1 expression in the mucosal surface could not serve as a biomarker for determining therapeutic efficacy [38]. Based on the heterogeneous results of this study, it was speculated that biopsy specimens might be inappropriate to qualify the status of PD-L1 in patients with gastric cancer. Thus, it is important to understand the basis for heterogeneous PD-L1 expression in gastric cancer in the future.

There are some limitations associated with this study. First, this was a single facility-based study with a small cohort. Second, PD-L1 expression was determined using 
a single antibody and did not consider the differences in staining potentials by multiple antibodies. Further, the study only focused on submucosal cancer owing to surgical resections. To preliminarily investigate PD-L1 expression in pT1a and pT2 cancers, we randomly stained 20 samples for each group, using our archived samples. We found that there was no difference in expression between the central portion and invasive front in $\mathrm{p}$ T1 a cases, while the expression of $\mathrm{PD}-\mathrm{L} 1$ in the invasive front was stronger than in the central portion in pT2 cases (data not shown). This may motivate further detailed studies on PD-L1 expression in the initial progressive stages. Thus, it is imperative to analyze samples from mucosal cancer obtained by endoscopy in the future to elucidate the mechanism(s) employed by early gastric cancer in evading immune responses.

In conclusion, $\mathrm{PD}$-L1-expressing cancer cells at the invasive front of tumors are important during the initial stages of invasion and lymphovascular permeation in early gastric cancer. Studying the immune checkpoint mechanisms employed during the initial phases of invasion may be a promising area of cancer research in the future.

\section{Statement of Ethics}

This research complies with the guidelines for human studies and was conducted ethically in accordance with the World Medical Association Declaration of Helsinki. Subjects (or their parents or guardians) have given their written informed consent. The study was approved by the Ethics Committee of Gunma University (approval No.: HS2019-183).

\section{Conflict of Interest Statement}

No potential conflicts of interest were disclosed.

\section{Funding Sources}

The authors declare that they received no funding support for this study.

\section{Author Contributions}

Conception and design of the study: Hiroyuki Kuwano, Ken Shirabe, Tetsunari Oyama, Hiroshi Saeki. Acquisition of data: Yasunari Ubukata, Yuki Shimoda, Tadashi Handa. Analysis and/or interpretation of data: Yasunari Ubukata, Kyoichi Ogata, Takehiko Yokobori. Drafting the manuscript: Yasunari Ubukata, Hiroshi Saeki. Revising the manuscript critically for important intellectual content: Kyoichi Ogata, Makoto Sohda, Takehiko Yokobori, Nobuhiro Nakazawa, Akiharu Kimura, Norimichi Kogure, Akihiko Sano, Makoto Sakai, Hiroomi Ogawa. Approval of the version of the manuscript to be published: Yasunari Ubukata, Kyoichi Ogata, Makoto Sohda, Takehiko Yokobori, Yuki Shimoda, Tadashi Handa, Nobuhiro Nakazawa, Akiharu Kimura, Norimichi Kogure, Akihiko Sano, Makoto Sakai, Hiroomi Ogawa, Hiroyuki Kuwano, Ken Shirabe, Tetsunari Oyama, Hiroshi Saeki.

\section{References}

1 Cancer Information Service, National Cancer Center, Japan. Cancer Registry and Statistics. 2019 Dec. Available from: https://www.ncc. go.jp/en/cis/index.html

2 Barreto SG, Windsor JA. Redefining early gastric cancer. Surg Endosc. 2016 Jan;30(1): 24-37.

3 Hölscher AH, Drebber U, Mönig SP, Schulte C, Vallböhmer D, Bollschweiler E. Early gastric cancer: lymph node metastasis starts with deep mucosal infiltration. Ann Surg. 2009 Nov;250(5):791-7.

4 Mönig S, Baldus SE, Collet PH, Zirbes TK, Bollschweiler E, Thiele J, et al. Histological grading in gastric cancer by Goseki classification: correlation with histopathological subtypes and prognosis. Anticancer Res. $2001 \mathrm{Feb} ; 21(1 \mathrm{~B}): 617-20$.

5 Luebke T, Baldus SE, Grass G, Bollschweiler E, Thiele J, Dienes HP, et al. Histological grading in gastric cancer by Ming classification: correlation with histopathological subtypes, metastasis, and prognosis. World J Surg. 2005 Nov;29(11):1422-7.
6 Bonenkamp JJ, Songun I, Hermans J, Sasako M, Welvaart K, Plukker JT, et al. Randomised comparison of morbidity after D1 and D2 dissection for gastric cancer in 996 Dutch patients. Lancet. 1995 Mar;345(8952):745-8.

7 Cuschieri A, Fayers P, Fielding J, Craven J, Bancewicz J, Joypaul V, et al. Postoperative morbidity and mortality after D1 and D2 resections for gastric cancer: preliminary results of the MRC randomized controlled surgical trial. Lancet. 1996 Apr;347(9007):995-9.

8 Lauwers GY, Carneiro F, Graham DY, Curado MP, Franceschi S, Montgomery E, et al. Gastric carcinoma. In: Bosman FT, Carneiro F, Hruban RH, Theise ND, editors. WHO Classification of Tumours of the Digestive System, 4th ed. Switzerland: World Health Organization; 2010. p. 48-58.

9 Oshima T, Shan J, Okugawa T, Chen X, Hori $\mathrm{K}$, Tomita T, et al. Down-regulation of claudin-18 is associated with the proliferative and invasive potential of gastric cancer at the invasive front. PLoS One. 2013 Sep;8(9):e74757.
10 Okugawa T, Oshima T, Chen X, Hori K, Tomita T, Fukui H, et al. Down-regulation of claudin-3 is associated with proliferative potential in early gastric cancers. Dig Dis Sci. 2012 Jan;57(6):1562-7.

11 Riley JL. PD-1 signaling in primary T cells. Immunol Rev. 2009 May;229(1):114-25.

12 Okazaki T, Honjo T. PD-1 and PD-1 ligands: from discovery to clinical application. Int Immunol. 2007 Jul;19(7):813-24.

13 Ribas A. Tumor immunotherapy directed at PD-1. N Engl J Med. 2012 Jun;366(26):25179.

14 Topalian SL, Hodi FS, Brahmer JR, Gettinger SN, Smith DC, McDermott DF, et al. Safety, activity, and immune correlates of anti-PD-1 antibody in cancer. N Engl J Med. 2012 Jun; 366(26):2443-54.

15 Brahmer JR, Tykodi SS, Chow LQ, Hwu WJ, Topalian SL, Hwu P, et al. Safety and activity of anti-PD-L1 antibody in patients with advanced cancer. N Engl J Med. 2012 Jun; 366(26):2455-65. 
16 Tsutsumi S, Saeki H, Nakashima Y, Ito S, Oki E, Morita M, et al. Programmed death-ligand 1 expression at tumor invasive front is associated with epithelial-mesenchymal transition and poor prognosis in esophageal squamous cell carcinoma. Cancer Sci. 2017 May;108(6): 1119-27.

17 Ohigashi Y, Sho M, Yamada Y, Tsurui Y, Hamada K, Ikeda N, et al. Clinical significance of programmed death-1 ligand-1 and programmed death-1 ligand-2 expression in human esophageal cancer. Clin Cancer Res. 2005 Apr;11(8):2947-53.

18 Umemoto Y, Okano S, Matsumoto Y, Nakagawara $\mathrm{H}$, Matono R, Yoshiya S, et al. Prognostic impact of programmed cell death 1 ligand 1 expression in human leukocyte antigen class I-positive hepatocellular carcinoma after curative hepatectomy. J Gastroenterol. 2015 Jan;50(1):65-75.

19 Thompson RH, Kuntz SM, Leibovich BC, Dong H, Lohse CM, Webster WS, et al. Tumor $\mathrm{B} 7-\mathrm{H} 1$ is associated with poor prognosis in renal cell carcinoma patients with long-term follow-up. Cancer Res. 2006 Apr;66(7):3381-5.

20 Hino R, Kabashima K, Kato Y, Yagi H, Nakamura M, Honjo T, et al. Tumor cell expression of programmed cell death-1 ligand 1 is a prognostic factor for malignant melanoma. Cancer. 2010 Apr;116(7):1757-66.

21 Ren X, Wu H, Lu J, Zhang Y, Luo Y, Xu Q, et al. PD1 protein expression in tumor infiltrated lymphocytes rather than PDL1 in tumor cells predicts survival in triple-negative breast cancer. Cancer Biol Ther. 2018 Feb;19(5): 373-80.

$22 \mathrm{Wu}$ Y, Cao D, Qu L, Cao X, Jia Z, Zhao T, et al. PD-1 and PD-L1 co-expression predicts favorable prognosis in gastric cancer. Oncotarget. 2017 Sep;8(38):64066-82.

23 Li J, Chen L, Xiong Y, Zheng X, Xie Q, Zhou $\mathrm{Q}$, et al. Knockdown of PD-L1 in human gastric cancer cells inhibits tumor progression and improves the cytotoxic sensitivity to CIK therapy. Cell Physiol Biochem. 2017 Feb; 41(3):907-20
24 Mimura K, Teh JL, Okayama H, Shiraishi K, Kua LF, Koh V, et al. PD-L1 expression is mainly regulated by interferon gamma associated with JAK-STAT pathway in gastric cancer. Cancer Sci. 2018 Jan;109(1):43-53.

25 Weinberg BA, Xiu J, Hwang JJ, Shields AF, Salem ME, Marshall JL. Immuno-oncology biomarkers for gastric and gastroesophageal junction adenocarcinoma: Why PD-L1 testing may not be enough. Oncologist. 2018 Oct; 23(10):1171-7.

26 Takada K, Toyokawa G, Okamoto T, Akamine T, Takamori S, Katsura M, et al. An immunohistochemical analysis of PD-L1 protein expression in surgically resected small cell lung cancer using different antibodies and criteria. Anticancer Res. 2016 Jul;36(7):3409-12.

27 Wang W, Wang K, Chen Z, Chen L, Guo W, Liao $P$, et al. Immunoclassification characterized by CD 8 and PD-L1 expression is associated with the clinical outcome of gastric cancer patients. Oncotarget. 2018 Feb;9(15): 12164-73.

28 Harada K, Dong X, Estrella JS, Correa AM, Xu Y, Hofstetter WL, et al. Tumor-associated macrophage infiltration is highly associated with PD-L1 expression in gastric adenocarcinoma. Gastric Cancer. 2018 Jan;21(1):31-40.

29 Koh YW, Han JH, Haam S, Jung J, Lee HW. Increased CMTM6 can predict the clinical response to PD-1 inhibitors in non-small cell lung cancer patients. Oncoimmunology. 2019 Jun;8(10):e1629261.

30 Thompson ED, Zahurak M, Murphy A, Cornish T, Cuka N, Abdelfatah E, et al. Patterns of PD-L1 expression and CD8 T cell infiltration in gastric adenocarcinomas and associated immune stroma. Gut. 2017 May;66(5): 794-801.

31 Wang Y, Zhu C, Song W, Li J, Zhao G, Cao H. PD-L1 expression and CD8+ T cell infiltration predict a favorable prognosis in advanced gastric cancer. J Immunol Res. 2018 May; 2018:4180517.
32 Gao Y, Li S, Xu D, Chen S, Cai Y, Jiang W, et al. Prognostic value of programmed death-1, programmed death-ligand 1, programmed death-ligand 2 expression, and CD8(+) T cell density in primary tumors and metastatic lymph nodes from patients with stage T1$4 \mathrm{~N}+\mathrm{M} 0$ gastric adenocarcinoma. Chin J Cancer. 2017 Jul;36(1):61.

33 Tauchi Y, Tanaka H, Kumamoto K, Tokumoto M, Sakimura C, Sakurai K, et al. Tumorassociated macrophages induce capillary morphogenesis of lymphatic endothelial cells derived from human gastric cancer. Cancer Sci. 2016 Aug;107(8):1101-9.

34 Burr ML, Sparbier CE, Chan YC, Williamson JC, Woods K, Beavis PA, et al. CMTM6 maintains the expression of PD-L1 and regulates anti-tumour immunity. Nature. 2017 Sep; 549(7670):101-5.

35 Shen B, Qian A, Lao W, Li W, Chen X, Zhang B, et al. Relationship between Helicobacter pylori and expression of programmed death-1 and its ligand in gastric intraepithelial neoplasia and early-stage gastric cancer. Cancer Manag Res. 2019 May;11:3909-19.

36 Okabe M, Toh U, Iwakuma N, Saku S, Akashi M, Kimitsuki Y, et al. Predictive factors of the tumor immunological microenvironment for long-term follow-up in early stage breast cancer. Cancer Sci. 2017 Jan;108(1):81-90.

37 Kohisa S, Oki E, Iimori M, Nakaji Y, Shimokawa $M$, Saeki $H$, et al. Clinical significance of programmed cell death-ligand 1 expression and the immune microenvironment at the invasive front of colorectal cancers with high microsatellite instability. Int J Cancer. 2018 Feb;142(4):822-32.

38 Kang YK, Boku N, Satoh T, Ryu MH, Chao Y, Kato K, et al. Nivolumab in patients with advanced gastric or gastro-oesophageal junction cancer refractory to, or intolerant of, at least two previous chemotherapy regimens (ONO4538-12, ATTRACTION-2): a randomised, double-blind, placebo-controlled, phase 3 trial. Lancet. 2017 Dec;390(10111):2461-71. 